\title{
Multipolar generator for lower-power windmills
}

\author{
A. Chuchalin, I. Safyannikov and I. Rossamakhin \\ Department of Electrical Engineering \\ Tomsk Polytechnic University \\ 30, Lenin av., 634050, Tomsk (Russia) \\ phone: (3822) 563-806, fax: (3822) 527-499, e-mail: chai@tpu.ru, refer@tpu.ru
}

\begin{abstract}
In this paper a new design of disk type multipolar electric generator with electromagnetic excitation is introduced. Applying the generator mentioned makes it possible to enhance the performance effectiveness of wind-driven power plants. The diagrammatic representation of an active zone presented serves as an example of generator design and operation principle. The basic distinguishing features of design and its position in the electrical machines classification are pointed out.
\end{abstract}

\section{Key words}

Wind-power engineering, wind-powered generator, energy efficiency, slow-speed windmill.

\section{Introduction}

Intensive development of electroenergetics based on utilizing natural renewed energy sources (water, wind) keeps one searching for new approaches in the area of electromechanical converts design [1]. It applies to a considerable extent to wind-power engineering and is connected with low density of energy carrier and instability of its parameters (wind velocity, its stability and air moisture). This results in lowering considerably the coefficient of energy conversion in wind-driven power plant (WDPP).

To solve the problem of enhancing radically the operation effectiveness of WDPPs is removing multiplicator from their composition. WDPP without reducer are of enhanced value of efficiency and reliability and lowered production cost as well. Special multipolar design of electric generator may be applied to implement the problem. Using multipolar electric generators makes it also possible to introduce more effective versions of WDPP with slow-speed types of operating wind turbines.

Wider acceptance of slow-speed wind plants is determined by the peculiarities of value distribution structure of average annual wind velocities on the territory of Russia. Now, the regions with average speed more than $4 \mathrm{~m} / \mathrm{sec}$ of long standing are admitted to be economically effective for using wind-power [2]. Basically, these are riversides and hills. The estimation mentioned is based on the possibilities and characteristic features of the most widely spread types of WDPP comprising multiplicator. Therefore, territorial enlargement for applying WDPP should be first connected with developing wind plants without reducer diagram of wind turbine and generator.

\section{Main part}

Analyzing the available designs of low speed machines makes it possible to distinguish two basic approaches for solving the problem. First, it is reducing angular dimension of pole pitch by applying magnetoelectric excitation system [3]-[5]. Second, increasing relative rotational speed of the machine active zone (birotatory design) [6] where permanent magnets are also supposed to be used.

Applying magnetoelectric system makes it possible to design the generators with operating rotational speed from tens to hundreds revolutions per minute [3]. Besides, generators on permanent magnets have many advantages. First, the lack of sliding contacts unit results in enhancing the level of generators reliability. Second, rotor constructions without windings is easy to manufacture and more reliable. Third, eliminating electric "excitation" loss increases the value of generator efficiency.

However, to provide the preset excitation level at low limit of the wind-turbine operating rotational speed range calls for applying high-coercivity rare-earth magnets. Caused by high cost of the magnets like these the cost of generator and unit as a whole rises. It's impossible to adjust the value of excitation flux in magneto-electric excitation system. The drawback like this contributes to applying more expensive power circuit for regulating the generator voltage amplitude. Thus, applying low speed 
generators with magnetoelectric excitation system is characterized by increasing the power unit cost and this is also ambiguous as to enhancing the effectiveness of functioning.

Besides, in the designs available the constructing principles of active zone characteristic of synchronous machines are more often used. Characteristic feature of the active zone is sequential arrangement of opposite pole pairs along rotor movement direction. (Fig. 1)

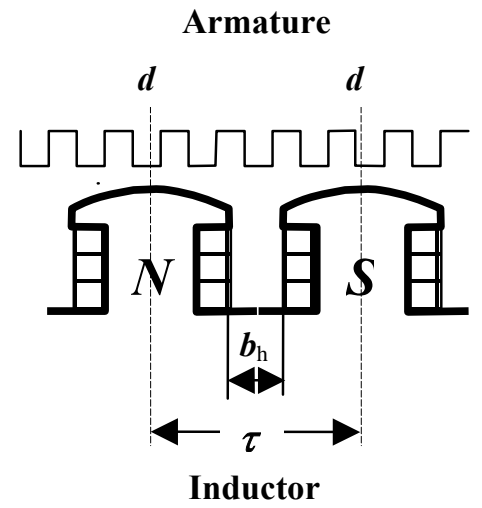

Fig. 1. Diagram of active zone in the synchronous machines.

By this, minimal value of interpole distance $\boldsymbol{b}_{\mathrm{h}}$ and pole pitch dimension $\boldsymbol{\tau}$ are limited as a whole. So, using synchronous machines structure for designing multipolar generator is limited by the designing features of the structure itself.

Applying the diagram of machine active zone with pulsating flow makes it possible to gain considerable lowering of the pole pitch size (Fig. 2). Pole system including the unidirectional magnetized poles is characteristic of inductor machines with homopolar. That is why, concentrated excitation winding common for all poles being arranged on the stationary part of the machine - stator is used. This winding arrangement doesn't influence the size of the teeth $\left(\boldsymbol{b}_{\mathrm{z}}\right)$ themselves and tooth pitch $\left(\tau_{\mathrm{z}}\right)$ and determines the fact that electromagnetic excitation machine is contactless. Besides, the phenomenon of interpole leakage flux is eliminated by their unidirectional magnetization.

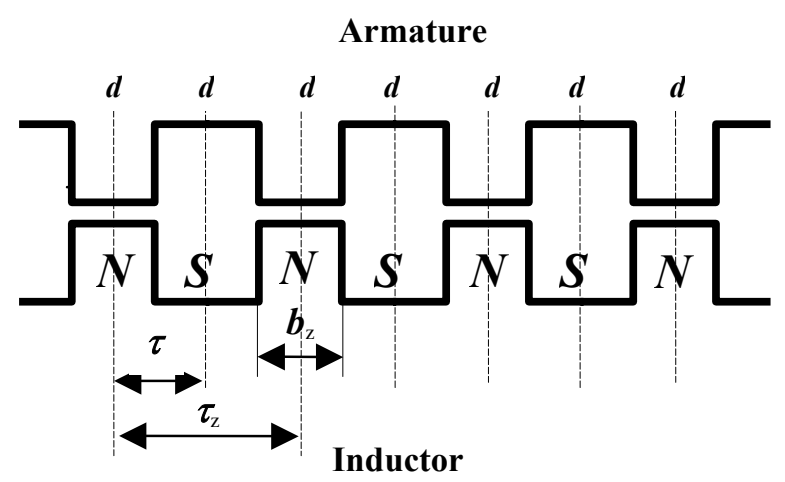

Fig. 2. Diagram of the active zone of pulsating type machine.
However, inductor machines found their basic application as high frequency power sources. Using them as low speed commercial frequency generators hasn't yet found their application. It is basically caused by principally low coefficient of utilizing the excitation flow $\boldsymbol{k}_{1}=\left(\Psi_{1 a f}^{\max }-\Psi_{1 \text { af }}^{\min }\right) /\left(2 \Psi_{1 \text { af }}^{\max }\right)$, the value of which doesn't exceed 0,35 (Fig. 3). This contributes to low machine efficiency and its specific power value $(\mathrm{kW} / \mathrm{kg})$.

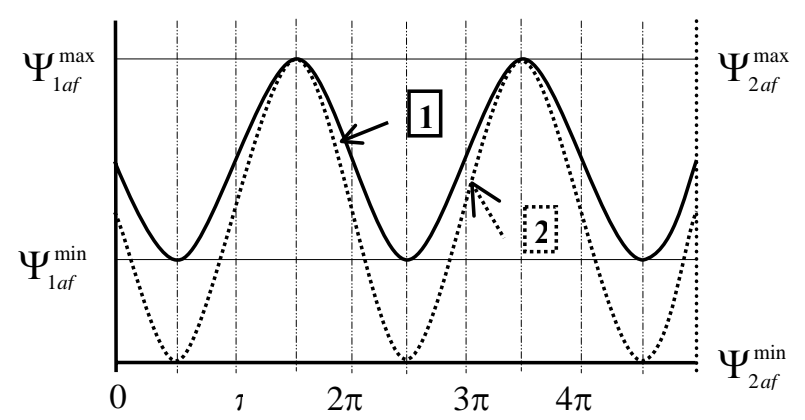

Fig. 3. Law of varying mutual flux linkage:

1 - for inductor homopolar machines,

2 - for one of winding multipolar switchboard generator.

Another variety of pulsating type machines is switchboard ones. Here, applying the principle of flux switching results in rising the coefficient of utilizing $\boldsymbol{k}_{1}$. Electromagnetic excitation system is also characteristic of these machines and they also belong to a group of contactless machines.

But the proportional connection between the pole pitch dimension and armature winding and excitation winding sizes is the drawback of these type machines.

The design of switchboard type multipolar generator considered in the paper combines all the merits of the machines with pulsating flow (Fig. 4). Similar to inductor homopolar generator the stationary excitation winding common for all poles is available in the generator mentioned. This makes it possible to considerably reduce the pole pitch dimension and causes the machine to be contactless. For measuring the flux in the air gap the flow switching principle is applied. By this, the coefficient of utilizing magnetic flux $\boldsymbol{k}_{2}=\left(\Psi_{2 a f}^{\max }-\Psi_{2 a f}^{\min }\right) /\left(2 \Psi_{2 a f}^{\max }\right)$ in comparison with inductor generators rises. As to operation principle the generator presented belongs to switchboard type machines [7].

The design of the generator active zone is schematically presented in Fig. 4. Generator stator comprises three units: central and two extreme ones separated from it by rotor disks through air gaps. Stator central unit is the generator excitation system and contains concentrated winding 1 with two groups of horseshoe laminations of core $\mathbf{2}$ and $\mathbf{3}$ enclosing it enters this unit. The laminations of these groups are arranged in turn and opposite to each other. Generator armature includes two magnetic and electric symmetric parts being biased relative to each other at angular pitch corresponding to one pole pitch. Similar to excitation system unit each of armature 
sections contains concentrated winding $\mathbf{5}$ and core $\mathbf{4}$ laminations enclosing it. The windings of both sections are connected in series. The laminations on each of the armature sections are arranged unidirectional. Generator rotor comprises shaft and two nonmagnetic disks 6. Each of the disks includes the laminations of the core $\mathbf{7}$ and $\mathbf{8}$ with two different directions functioning as switches of magnetic flux circuits. Rotor disks are also magnetically symmetric relative to each other.

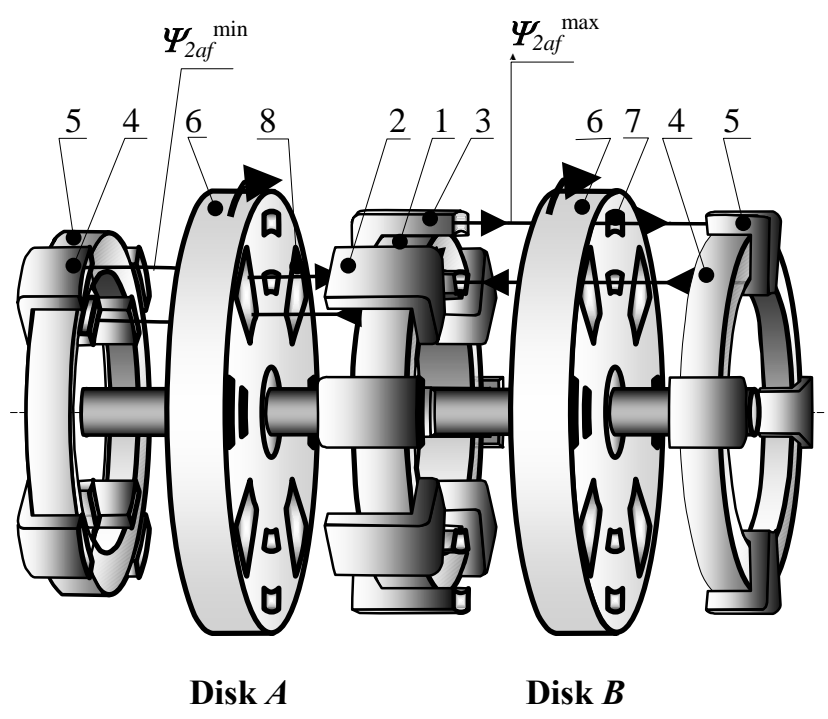

Fig. 4. Active zone of multipolar switchboard disk type generator.

Mutual position of core $\mathbf{7}$ and $\mathbf{8}$ laminations at the rotor $\boldsymbol{A}$ and $\boldsymbol{B}$ disks (Fig. 4) ensures maximal value of flux linkage between excitation winding in the right section of an armature $\Psi_{2 a f}^{\max }$ through the laminations 7 of rotor $\boldsymbol{B}$ disk. Equally mutual flux linkage of excitation winding with left section of armature winding is minimal owing to shunting action of laminations $\mathbf{8}$ on the rotor $\boldsymbol{A}$ disk. At subsequent angular rotor position the values of mutual flux linkages for the windings on the armature sections change into opposite one. Shunting excitation magnetic field results in lowering considerably the value of flux linkage $\Psi_{2 a f}^{\min }$. Compared to inductor machines multipolar switchboard generator has an enhanced up to $\boldsymbol{k}_{2}=0,5$ value of coefficient of utilizing excitation flow (Fig. 3). The generator physical model has been built up for confirming qualitatively the effect achieved.

Axial direction of excitation flow in an air gap, concentrated type of armature and excitation winding and distributed type of magnetic system may be attributed to distinguishing features of this design.
Total combination of the features mentioned makes it possible to considerably reduce relative value of pole pitch $\boldsymbol{\tau}$ and manufacturing cost of active zone. This is achieved by simplifying the manufacturing technology of the core and windings. The core is manufactured as to wasteless technology by winding electrical steel band. Concentrated coil winding manufacturing is the simplest one.

\section{Conclusion}

The design of contactless multipolar generator presented with the switchboard generator properties (flux switching) maintains the basic merit of inductor type generators - their multipolar features. Applying multipolar generator makes it possible to use without reducer diagram for building WDPP. This in combination with the contactless features of electromagnetic excitation generator will enhance the efficiency and WDPP reliable operation.

Lowering the generator unit cost (USD/kW) is achieved by simplifying the manufacturing technology of active zone.

\section{Acknowledgement}

The authors thank the Russian Education Ministry for partial support of this work.

\section{References}

[1] A.F. Diyakov, "Trends development of the renewable energy in Russia”, Izv. Academies of the Sciences, Russian J. Energy, No. 4, 2002, pp. 13-29.

[2] I.Yu. Ivanov, T.F. Tuguzova, S.P. Popov and N.A. Petrov, The North small electrical engineering. Problems and development trends, Russian Science, Novosibirsk (2002), $188 \mathrm{p}$.

[3] A.F. Shevchenko, "Multipolar magnetoelectric generators with fractional one-tooth windings. Russian J. Electrical engineering, No. 9, 1977, pp. 13-16.

[4] Ya.B. Danilevitch and A.V. Kochenev, "Synchronous low power generator with permanent magnets", Russian J. Electricity, No. 4, 1996, pp. 27-29.

[5] A.A. Afanasiev and A.V. Nikolaev, "Mathematical model of electrical generator with permanent magnets for wind plant”, Bulletin of the Urals State technical university, No. 5 part 2, 2003, pp. 32-35.

[6] B.V. Litvinov, "Single-phase synchronous double rotational generator excited from high power permanent magnets", Russian J. Electrical engineering, No. 4, 1998, pp. 20-25.

[7] D.A. But, "Modifications of switchboard increased voltage generator”, Russian J. Electricity, No.3, 1982, pp. 31-35. 